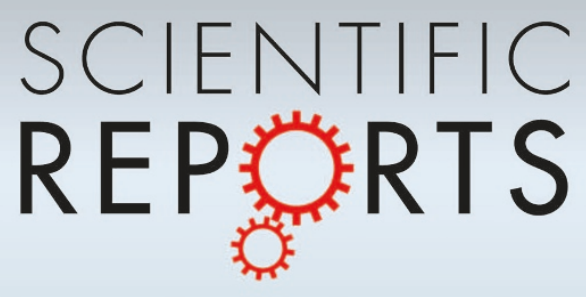

\title{
OPEN Active transport of vesicles in neurons is modulated by mechanical tension
}

SUBJECT AREAS:

BIOLOGICAL PHYSICS

BIOPOLYMERS IN VIVO

TRANSPORTERS IN THE NERVOUS

SYSTEM

\author{
Wylie W. Ahmed* \& Taher A. Saif \\ Department of Mechanical Science and Engineering, University of Illinois, Urbana, IL 61801.
}

NANOSCALE BIOPHYSICS

Received

9 December 2013

Accepted

24 February 2014

Published

27 March 2014

Effective intracellular transport of proteins and organelles is critical in cells, and is especially important for ensuring proper neuron functionality. In neurons, most proteins are synthesized in the cell body and must be transported through thin structures over long distances where normal diffusion is insufficient. Neurons transport subcellular cargo along axons and neurites through a stochastic interplay of active and passive transport. Mechanical tension is critical in maintaining proper function in neurons, but its role in transport is not well understood. To this end, we investigate the active and passive transport of vesicles in Aplysia neurons while changing neurite tension via applied strain, and quantify the resulting dynamics. We found that tension in neurons modulates active transport of vesicles by increasing the probability of active motion, effective diffusivity, and induces a retrograde bias. We show that mechanical tension modulates active transport processes in neurons and that external forces can couple to internal (subcellular) forces and change the overall transport dynamics.

Correspondence and requests for materials should be addressed to

T.A.S. (saif@illinois.

edu)

* Current address: Laboratoire PhysicoChimie Curie (UMR 168), Institut Curie, Paris, France 75231

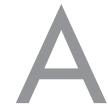

ctive transport is critical in maintaining biological functions in living cells. ${ }^{1}$. This is especially true in neurons where axons and dendrites have long aspect ratio geometry, which limits the effectiveness of passive diffusion. Cargo transport in cells is mediated by a stochastic interplay of passive diffusion and active transport ${ }^{2}$. Passive diffusion occurs when particles are moving randomly through the viscoelastic subcellular space ${ }^{3}$. Passive behavior resembles Brownian motion where the mean squared displacement is proportional to time $(\mathrm{MSD} \propto D \tau)$. Active transport is directed motion along cytoskeletal structures that is driven by molecular motors ${ }^{4}$. Active behavior resembles directed motion where the mean squared displacement is proportional to the square of time (MSD $\propto V^{2} \tau^{2}$ ). Thus by measuring how the MSD of vesicles scales with time it is possible to determine their mode of transport ${ }^{5,6}$. Fig. la,b shows a representative image and a schematic of a vesicle switching between active transport along a microtubule and passive diffusion in the subcellular space. This process allows the neuron to control the spatial organization of vital proteins and molecules throughout its complex structures. As an example, if a synaptic protein is synthesized in the cell body, it may need to be transported the entire length of the axon (which could be over 1 meter in a human) to reach its functional target. Thus active transport of specific subcellular cargo can be used to target different locations in the neuron?

Investigating the mechanisms of neuronal transport is critical in understanding neuronal function. Proper transport of vesicles and their cargo to specific locations in the cell is critical in building and maintaining synaptic machinery as well as modulating synaptic plasticity. For instance, preassembled units of synaptic proteins are transported in vesicles to synapses to provide building blocks for the active zone, which is necessary for rapid fusion of synaptic vesicles ${ }^{7}$. And activity-dependent synaptic plasticity involves rapid recruitment (under $10 \mathrm{~min}$ ) of synaptic vesicles associated with synaptophysin to the presynaptic terminal ${ }^{9}$. Additionally, a deficit in neuronal transport is an early pathogenic event and possibly the cause of several neurodegenerative diseases ${ }^{10,11}$.

Mechanical tension exists in neurons and stretch growth of axons is critical during developmental stages ${ }^{12-14}$ Recently tension has been implicated in maintaining normal vesicle dynamics ${ }^{6,15,16}$, but the underlying mechanism is not well understood. The relationship between neuronal stretch and vesicle transport has not been directly investigated. To this end, we analyzed active transport of endogenous large dense core vesicles of in vitro Aplysia neurons under external mechanical strain, and quantified their dynamics using tools from statistical physics. We used a stretchable substrate to apply tensile or compressive strain to cultured Aplysia neurons ${ }^{17}$ and recorded vesicle dynamics via high-speed video microscopy. Vesicle trajectories were tracked and analyzed using a temporal Mean Squared Displacement $(t \mathrm{MSD})$ analysis to quantify active and passive transport ${ }^{6}$. Active vesicle motion was used to investigate directed motion driven by molecular motors and passive motion was used to estimate the effective diffusivity of vesicles in the cytoplasm. 
(a)

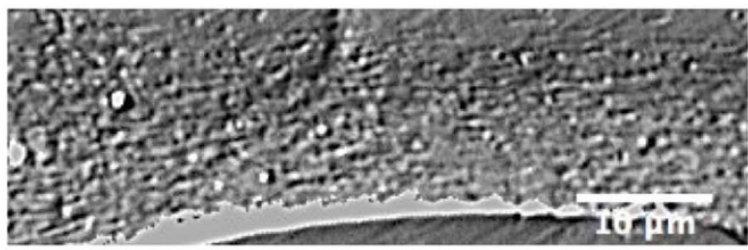

(b)
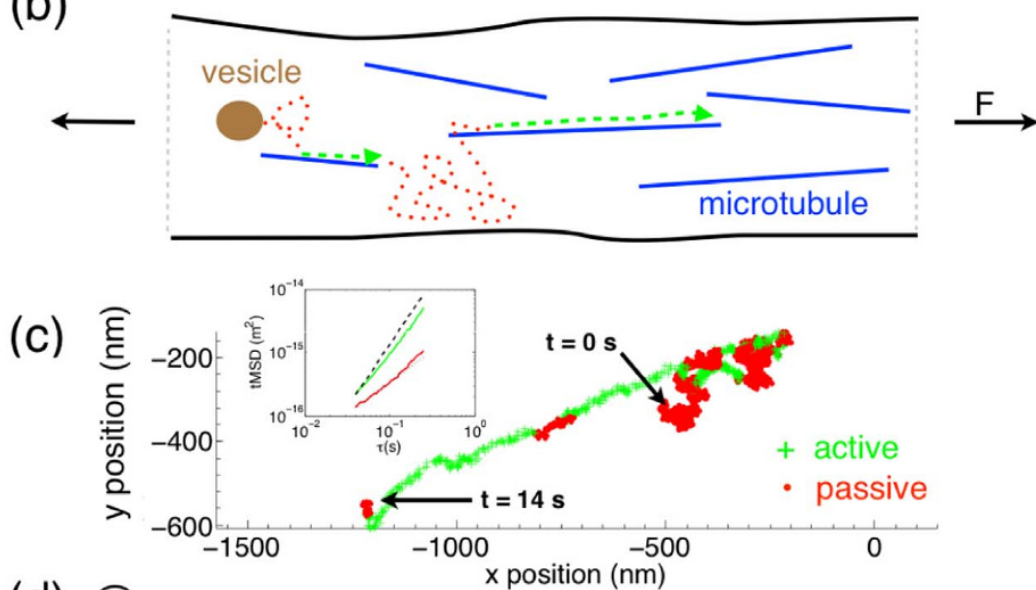

(d)

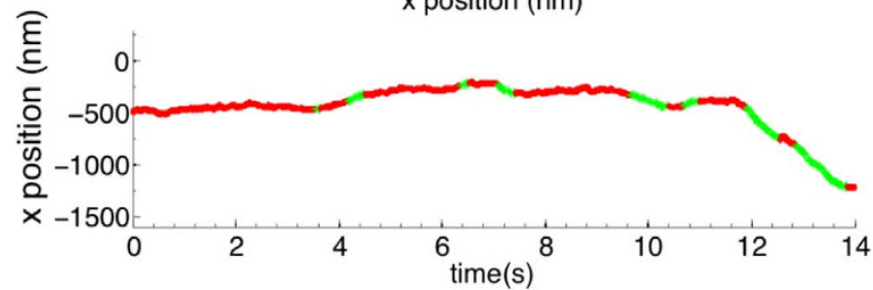

(e)

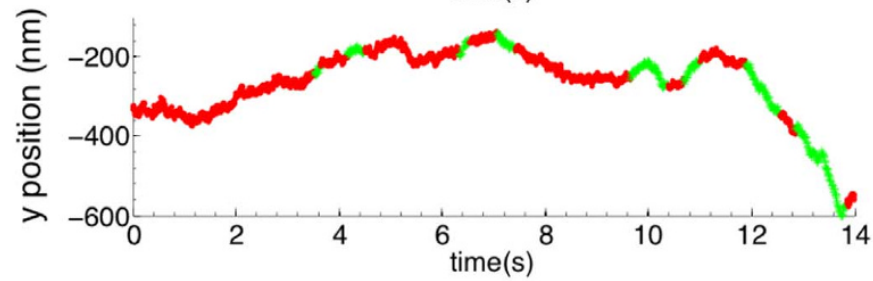

Figure 1 Vesicles switch stochastically between active and passive transport states. (a) A representative image of an Aplysia neurite showing vesicles of varying size (example image sequence available in supplementary information, video S1). (b) Simplified schematic of vesicle transport in a neurite. Vesicles alternate between active transport along microtubules and passive brownian-like motion. Mechanical strain is applied to modulate tension along neurite length and vesicle dynamics are tracked. (c) Plot of a representative trajectory, where the $t$ MSD algorithm is used to determine active ( $\alpha \geq 1.4)$ and passive motion $(\alpha<1.4)$. This example clearly shows the vesicle switching between active and passive behavior. The inset shows the slope of the average MSD for active (green) and passive (red) vesicle motion for the trajectory shown. (d,e) Plots of the $x$ and $y$ position as a function of time show that particle behavior is passive (red) most of the time and that when vesicles undergo active motion (green) they are moving over larger distances.

\section{Results}

Measuring active transport of vesicles. Vesicle motion is characterized as active or passive depending on the persistent directionality of its trajectory. To quantify vesicle dynamics the $t$ MSD method is used as described previously ${ }^{6}$. A representative image, schematic diagram, and experimental data of the motion of a vesicle in a neurite is shown in Fig. 1 (see supplementary video S1). The $t$ MSD analysis gives a measure of the distance a vesicle travels from an initial location during time $\tau$, and $\alpha$ is the slope of the $\operatorname{MSD}(\tau)$. $\alpha=1$ for Brownian motion, and $\alpha=2$ for a vesicle moving at a constant speed. The $t$ MSD can be plotted for the vesicle at any time during its journey as a means to investigate whether it is moving actively or passively by evaluating its $\alpha$ at that time. In our analysis, we use $\tau$ on the interval 100-160 ms to determine $\alpha$. Furthermore, we consider motions with $\alpha \geq 1.4$ as active based on calibrations from experiments and Brownian simulations.

The measured trajectory of a vesicle is color coded to illustrate the stochastic switching between active ( $\alpha_{\text {active }} \geq 1.4$, green) and passive $\left(\alpha_{\text {passive }}<1.4\right.$, red) states (Fig. 1c-e). This experimental data is an example showing that a vesicle undergoing active transport moves directionally and over longer distances compared to vesicles undergoing passive motion. In addition, when a vesicle is being actively transported it moves at a higher velocity as indicated by the steeper slope of the green data points (Fig. 1d,e). It should be noted that Aplysia bag cell neurons have vesicles ranging in size from $30 \mathrm{~nm}$ to nearly $1 \mu \mathrm{m}^{18}$. Vesicles were defined as large or small based on the median vesicle size $r \approx 350 \mathrm{~nm}$ measured from the images, however size determination of many tracked vesicles may be diffraction limited.

Probability of active motion increases due to stretch. To quantify the amount of active transport the probability of active motion, $P_{\mathrm{a}}$, is estimated from each image sequence by dividing the time of active vesicle motion, $t_{\text {active }}$, by the total tracked time, $t_{\text {total }}$,

$$
P_{\mathrm{a}}=\left\langle\frac{t_{\text {active }}}{t_{\text {total }}}\right\rangle
$$


where $\langle\cdots\rangle$ indicates an average over all cells and vesicles. In control neurons (Fig. 2, red), the probability of active motion is steady and remains $\sim 0.09$. This value is similar to the probability of active motion observed for tracer beads in a remodeling actin-myosin gel $^{19}$. To create a remodeling gel, Stuhrmann et al. ${ }^{19}$ reconstituted a network of actin with cross-links and myosin II filaments, resulting in a gel driven out of equilibrium by polymerizing actin and myosin motors. The myosin motors move actin filaments that interact nonspecifically with tracer beads giving rise to active motion. As the gel network continues to remodel it approaches an equilibrium state where tracer beads no longer exhibit active motion, but instead resemble stationary beads in an elastic gel ${ }^{19}$. In the neuron the subcellular structure is relatively stable and significant reorganization is not expected, yet it maintains dynamics similar to a far from equilibrium remodeling actin-myosin gel. This may be due to the fact that vesicles in the neurons are being driven directly by molecular motors whereas tracer beads in the actin-myosin gel are moving via nonspecific interactions.

In stretched neurons (Fig. 2, blue), $P_{\mathrm{a} \text {,stretch increases sharply and }}$ peaks at $\sim 0.18$ after $25 \mathrm{~min}$ of stretch. Interestingly, after $25 \mathrm{~min}$, $P_{\mathrm{a} \text {,stretch }}$ begins to decay for large vesicles $\left(r_{\text {large }}>350 \mathrm{~nm}\right)$ but remains elevated for small vesicles $\left(r_{\text {small }}<350 \mathrm{~nm}\right)$. In compressed neurons, $P_{\mathrm{a} \text {,compress }}$ decreases slightly to $\sim 0.07$ for vesicles of all sizes and remains low for the duration of the experiment (Fig. 2, green). It is interesting that in all cases the small vesicles $(X)$ consistently have a higher probability of active motion when compared to large vesicles $(\square)$. It is possible that the smaller vesicles experience less resistance to motion compared to large vesicles since they may encounter fewer obstacles due to their decreased size. Overall, this data suggests that vesicles spend more time undergoing active transport when a neuron is stretched.

The relation between active transport of vesicles and mechanical stretch may be due to alterations in microtubule structure. Mechanical stretching may stabilize microtubules ${ }^{43}$ and compression (or stretch release) may induce bending and breakage ${ }^{44}$. This structural change could modify the kinetic on and off rates of molecular motors leading to a change in active transport. In addition, the slow change (20 $\mathrm{min}$ ) in probability of active motion may be due to signal transduction cascades induced by stretch activated ion channels ${ }^{45}$.

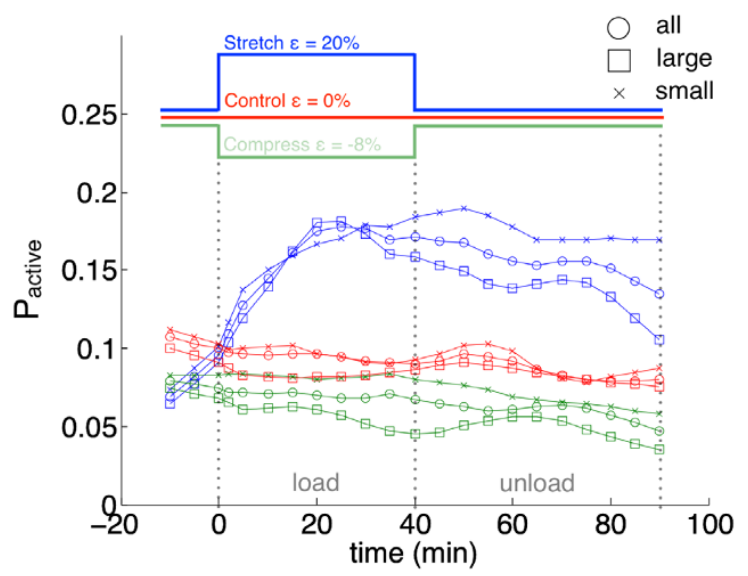

Figure $2 \mid$ Active transport of vesicles increases due to stretch. Probability of active motion of vesicles in control (red) neurons exhibit stable behavior with $P_{\mathrm{a} \text {,control }} \approx 0.09$. Under stretch (blue), vesicles exhibit more active motion, which peaks at $P_{\text {a,stretch }} \approx 0.18$ after $25 \mathrm{~min}$. The activity of small $(X)$ vesicles remains high whereas large $(\square)$ vesicle activity decreases. Vesicles in compressed (green) neurons exhibit slightly decreased activity relative to the control (red). [control (red), stretch (blue), compress (green), all vesicles $(\bigcirc)$, large vesicles $(\square)$, small vesicles $(X)$ ]. Solid color-coded lines indicate loading profile of experiments. $(n>19$ animals, $>200$ vesicles per cell).
Effective diffusion of vesicles increases due to stretch. By quantifying how the velocity of a specific vesicle changes over time, it is possible to estimate its effective diffusion coefficient. One method of quantification is to look at the velocity autocorrelation function of a vesicle, which provides insight on how the vesicle is interacting with its surrounding environment ${ }^{20}$. The normalized velocity autocorrelation function, $\psi(\tau)$, is defined as

$$
\psi(\tau)=\frac{\left\langle\mathbf{v}\left(t_{0}\right) \cdot \mathbf{v}\left(t_{0}+\tau\right)\right\rangle}{\left\langle\left[v\left(t_{0}\right)\right]^{2}\right\rangle}
$$

where $\mathbf{v}$ is the vesicle velocity, and $t_{0}$ is an arbitrary point in time. For a completely non-interacting system where vesicles move at constant speeds independent of $\tau$, and $\left\langle\mathbf{v}\left(t_{0}\right) \cdot \mathbf{v}\left(t_{0}+\tau\right)\right\rangle=\left\langle\left[v\left(t_{0}\right)\right]^{2}\right\rangle$, we find $\psi(\tau)=1$. This is not expected in a biological environment due to interactions between the vesicle and the crowded subcellular environment, and $\psi(\tau)$ exhibits a decay. Thus the rate of decay of $\psi(\tau)$ is a measure of how quickly the vesicle velocity decorrelates. Accordingly, the self diffusion coefficient can be evaluated from the integral of the velocity autocorrelation function, $D_{\mathrm{s}}=v_{0}^{2} \int_{0}^{\infty} \psi(\tau) \mathrm{d} \tau$, which is known as a special case of the Green-Kubo relations for obtaining transport coefficients ${ }^{20}$.

We choose a subset of vesicles that undergo primarily passive dynamics $(\alpha<1.4)$ over long periods (30 s) of time to quantify $\psi(\tau)$ and evaluate $D_{\mathrm{s}}$. More than $90 \%$ of the $30 \mathrm{~s}$ time, each vesicle undergoes passive motion. We assume that during this Brownian type motion, the vesicles are not driven by any motors and that the media around them is homogeneous and isotropic. In control neurons, $\psi(\tau)$ shows a characteristic decay and a self diffusion coefficient, $D_{\text {s,control }} \approx 2 \times 10^{-3} \mu \mathrm{m}^{2} / \mathrm{s}$ (Fig. 3a). When the neuron is stretched, $\psi(\tau)$ shows a slower decay, indicating less resistance to motion and a higher self diffusion coefficient, $D_{\mathrm{s} \text {,stretch }} \approx 5 \times$ $10^{-3} \mu \mathrm{m}^{2} / \mathrm{s}$ (Fig. 3b). Under compression, the behavior of $\psi(\tau)$ decays slightly more rapidly than the control, and $D_{\text {s,compress }} \approx$ $1 \times 10^{-3} \mu \mathrm{m}^{2} / \mathrm{s}$. These results show that the self diffusivity of the vesicle increases when the neuron is stretched, and decreases due to compression. Interestingly, this may suggest that a microstructural change has occurred during stretching that allows vesicles to move with less resistance.

Vesicle mobility increases due to stretch. We now can estimate the mobility of vesicles during active and passive motion. During passive motion, the thermal motion of the vesicles is resisted by drag from the cytoplasmic environment. A balance of thermal and drag forces gives $D_{\mathrm{s}}=\mu k_{\mathrm{B}} T^{20}$, where the mobility is $\mu=V / F$ (velocity/drag resistance). From our measured $D_{\mathrm{s}}$, we get an effective $\mu=D_{\mathrm{s}} / k_{\mathrm{B}} T$ for the passive states as $\mu_{\text {control }}=0.5 \mu \mathrm{m} / \mathrm{pN} \cdot \mathrm{s}$ in control neurons, $\mu_{\text {stretch }}=1.25 \mu \mathrm{m} / \mathrm{pN} \cdot \mathrm{s}$ in stretched neurons, and $\mu_{\text {compress }}=$ $0.25 \mu \mathrm{m} / \mathrm{pN} \cdot \mathrm{s}$ in compressed neurons. Thus, vesicle mobility increases by 2.5 times when the neurons are stretched compared to control. Under compression, mobility decreases by half.

Overall vesicle motion increases due to stretch. One method to quantify the overall motion of the vesicles is to calculate the average mean squared displacement, $\operatorname{MSD}(\tau)=\left\langle|r(t+\tau)-r(t)|^{2}\right\rangle_{0<t<t_{\max }}$, where $\langle\cdots\rangle$ indicates an average over all available time steps and all vesicles. Fig. $4 \mathrm{a}$ indicates the average MSD behavior in control neurons which is relatively constant in time. When neurons are stretched, the overall motion of vesicles increases significantly exhibited by an increase in the MSD. This effect is greatest at $t \sim$ $25 \mathrm{~min}$ after stretch has been applied, and is most noticeable at large timescales, $\tau>10 \mathrm{sec}$ (Fig. 4b). Conversely, when neurons are compressed (Fig. 4c), the MSD decreases, exhibiting significantly decreased motion for all timescales, $\tau$. 

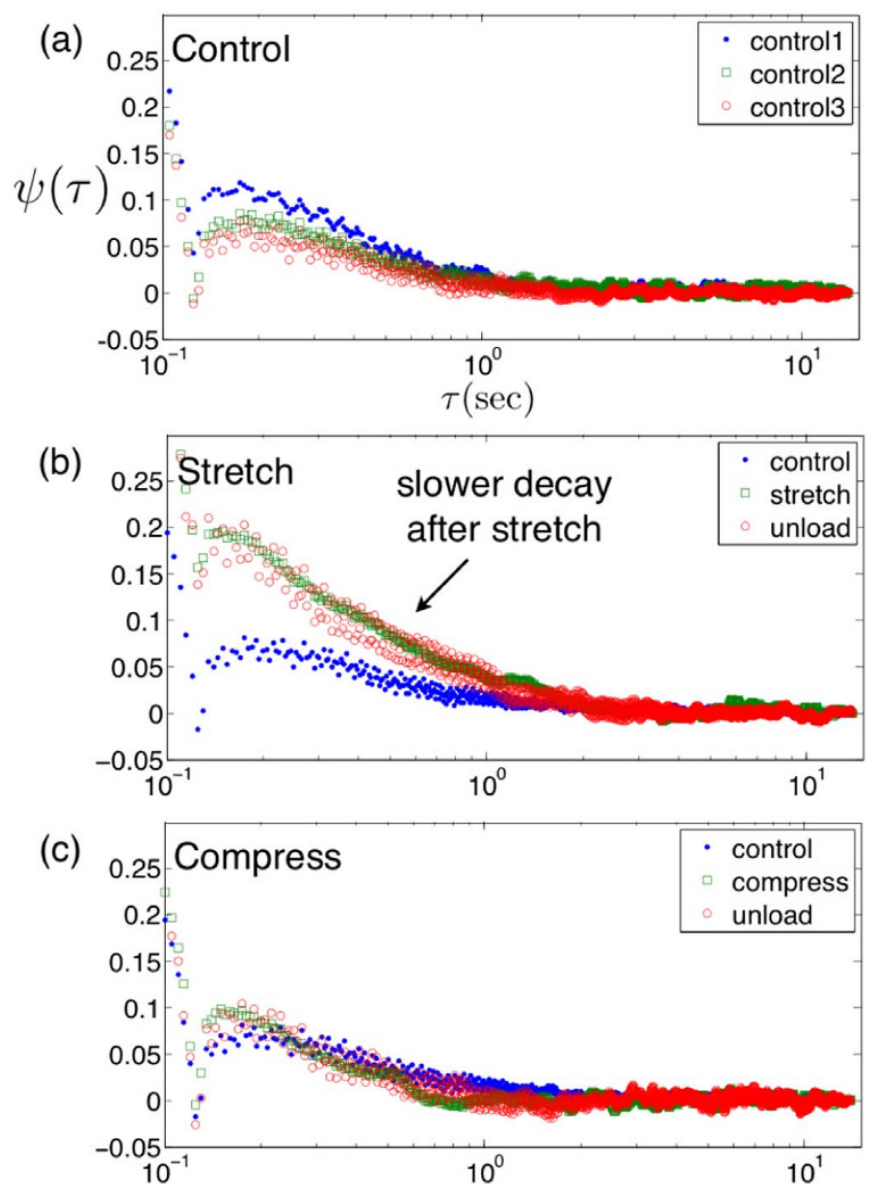

Figure 3 Self diffusivity of passive vesicles increases due to stretch. (a) Vesicles in control neurons exhibit a characteristic decay of $\psi(\tau)$ and $D_{\mathrm{s} \text {, control }} \approx 2 \times 10^{-3} \mu \mathrm{m}^{2} / \mathrm{s}$. (b) When neurons are stretched, vesicles exhibit a slower decay of $\psi(\tau)$ and thus have a higher estimated self diffusion coefficient of $D_{\mathrm{s} \text {,stretch }} \approx 5 \times 10^{-3} \mu \mathrm{m}^{2} / \mathrm{s}$. (c) Under compression, vesicles in neurons exhibit behavior similar to the control case. The estimated self diffusion coefficient is slightly lower, $D_{\mathrm{s} \text {, compress }} \approx 1 \times$ $10^{-3} \mu \mathrm{m}^{2} / \mathrm{s}$. Here, a subset of vesicles that maintain passive motion for most of the time span used ( $\sim 30 \mathrm{~s})$ are considered in the analysis.

Molecular motor activity increases due to stretch. To investigate the dynamics of vesicle motion it is useful to look at the distribution of vesicle displacements as a function of timescale. This is calculated by first choosing a timescale, $\tau$, and then building a frequency histogram of $\Delta x(\tau)$. From this we may calculate the probability distribution of vesicle displacements,

$$
P(\Delta x, \tau), \text { where } \Delta x(\tau)=x(t+\tau)-x(t),
$$

and by normalizing the histogram such that $\int_{-\infty}^{\infty} P(\Delta x, \tau) \mathrm{d} \Delta x=1$. The probability distribution, $P(\Delta x, \tau)$, also known as the Van Hove Correlation function (VHC), is a powerful tool for interpreting vesicle dynamics. The shape of the VHC can provide information on the nonequilibrium dynamics of the system ${ }^{21}$.

The VHC for particles undergoing Brownian motion in a fluid remains Gaussian at all times $\tau^{21}$, although the width of the Gaussian distribution may increase with $\tau$. However, if a small fraction of the particles in the ensemble occasionally take longer athermal jumps due to active transport (for example, by an external agent), then the histogram of $\Delta x(\tau)$ shows deviation from Gaussian ${ }^{22}$. For example, in active actin-myosin gels, the VHC of embedded probe particles exhibits a broader Gaussian regime and marked non-Gaussian tails ${ }^{19}$. The broader Gaussian region has been attributed to the collective action of many motors ${ }^{19}$, and the non-Gaussian tails suggest the action of a single motor ${ }^{22}$. For a passive actin gel, the VHC is expected to be mainly Gaussian ${ }^{22}$.

The results of the calculated VHC's and Gaussian fits of vesicle displacements are shown in Fig. 5, where active and passive behavior were separated using the $t$ MSD analysis $\left(\alpha_{\text {active }} \geq 1.4\right.$ and $\alpha_{\text {passive }}<$ $1.4)^{6}$. Here, we again choose subsets of vesicles that undergo primarily passive and primarily active states. Vesicles of each subset spend more than $90 \%$ of the time in their respective mode of motion (active or passive). The statistics of the Gaussian fit are shown as $\mu=\bar{x} \pm \sigma$. A non-zero mean, $\bar{x}$, of the distribution indicates a bias in vesicle motion. The standard deviation, $\sigma$, is a measure of the Gaussian width and is indicative of collective molecular motor activity ${ }^{19}$. A non-Gaussian parameter is defined as,

$$
\xi=\frac{\left\langle\Delta x(\tau)^{4}\right\rangle}{3\left\langle\Delta x(\tau)^{2}\right\rangle^{2}}-1
$$

which is a dimensionless parameter that is zero for a Gaussian distribution but takes on non-zero values to characterize deviation from Gaussianity, and provides a measure of single motor activity ${ }^{22}$. The VHC functions are plotted where the subscript "a" indicates active vesicles and the subscript "p" indicates passive vesicles (Fig. 5).

Overall, in all experimental conditions and at all timescales observed, $\sigma_{\mathrm{a}}>\sigma_{\mathrm{p}}$ and $\xi_{\mathrm{a}}<\xi_{\mathrm{p}}$. The larger Gaussian width, $\sigma_{\mathrm{a}}$, observed in active motion suggests active transport is due to a collective ensemble of many molecular motors ${ }^{19}$. The larger nonGaussian parameter, $\xi_{\mathrm{p}}$, observed in passive motion suggests that a few vesicles are undergoing occasional athermal jumps, possibly from single molecular motors ${ }^{22}$. The duration of such motions (jumps) is shorter than the time scale (160 ms in the $t$ MSD analysis) used to identify the state of their dynamics, and thus the vesicles become labelled as passive.

When neurons are stretched, $\xi_{\text {stretch }}>\xi_{\text {control }}$ (for $\tau=0.15$ and $1 \mathrm{sec}$ ), suggesting increased activity of single molecular motors. In addition, $\sigma_{\text {stretch }}>\sigma_{\text {control }}$ (for $\tau=1$ and $5 \mathrm{sec}$ ), suggesting higher activity of molecular motors giving rise to greater vesicle motion. It is worth noticing, that for stretched neurons the VHC at $\tau=1 \mathrm{sec}$ (Fig. $5 b$, zoomed inset) exhibits a local minima at $\Delta x \approx 0$, indicating that active vesicles are more likely to move than stay stationary. When neurons are compressed, they exhibit the opposite behavior: decreased motor behavior at all timescales. This result suggests that molecular motor activity increases in neurons due to stretch, which has been hypothesized to lead to significantly enhanced reaction kinetics in axonal structures ${ }^{23}$.

Mechanical stretch induces a retrograde bias in vesicle motion. Control neurons show no significant bias in vesicle motion $\left(\tilde{x}_{\text {control }} \approx 0\right)$, but when neurons are stretched they show a retrograde bias of vesicle motion $\left(\bar{x}_{\text {stretch }}=-26 \mathrm{~nm}\right)$ at larger timescales. This is most clear in the stretched neuron at $\tau=5 \mathrm{sec}$ (Fig. 5b), where the VHC is clearly not symmetric and the distribution has a negative mean, indicating a bias towards retrograde movements. To quantify the bias we estimate the probability of retrograde motion as, $p_{\mathrm{r}}(\tau)=\int_{-\infty}^{0} P(\Delta x, \tau) \mathrm{d} \Delta x$. In all experimental conditions, except stretched neurons, we see no significant bias, $p_{\mathrm{r}} \approx 0.5$. However, for stretched neurons we see $p_{\mathrm{r} \text {,stretch }}(\tau=5 \mathrm{~s})=0.59$ for active vesicles, indicating there is a significantly larger probability for vesicles to move in a retrograde direction. The retrograde bias emerges at long timescales, suggesting it is a result of coordinated activity of many molecular events. In axons, retrograde motion of the cargo is due to dynein motors (moving towards the $(-)$ end of the microtubules). Since, vesicles are moved by a cooperative activity of both dynein and kinesin motors engaged on the same vesicle ${ }^{1}$, a net retrograde motion of vesicles in stretched axons suggests a shift in the balance between dynein and kinesin. 
(a) Control

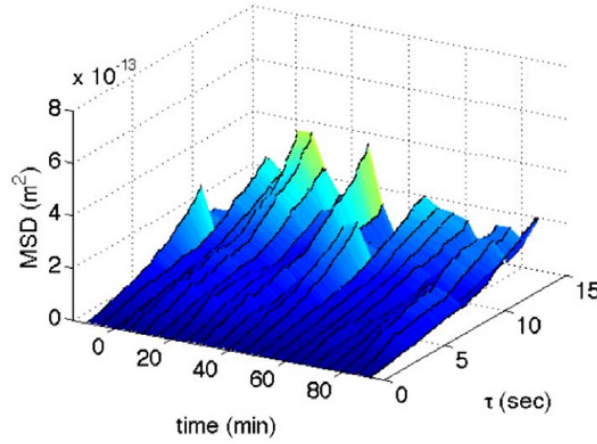

(b) Stretch

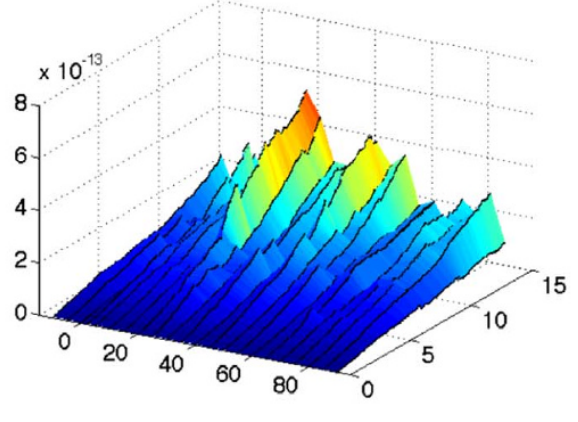

(c) Compress

Figure $4 \mid$ Overall motion of vesicles increases due to stretch. (a) Control neurons indicate normal behavior of vesicle motion in neurons over available timescales. (b) When neurons are stretched, the MSD increases significantly and is most noticeable after 25 min of applied stretch and at large timescales, $\tau$. (c) When compressed, the vesicle MSD decreases significantly immediately after compression and remains decreased for the duration of the experiment.

The dependence of $\mu$ and $\xi$ on timescale is expected because of the type of phenomenon being measured. The bias and collective activity (measured by $\bar{x}$ and $\sigma$ respectively) of vesicle motion are processes that require a large number of molecular interactions. Thus their behavior emerges over longer periods of time. The activity of single molecular motors (measured by $\xi$ ) are single (short) events which are evident at short timescales, but are averaged out at over longer timescales. Hence, changes in $\mu$ are more noticeable at long timescales, and $\xi$ at short timescales.

\section{Discussion}

Mechanical tension in neurons has been implicated in the processes of growth, development, and signaling ${ }^{24-26}$. Normal vesicle transport plays critical roles in many different processes. Here we will briefly discuss two topics: neurodegenerative disease and synaptic machinery.

Deficits in axonal transport are early pathogenic symptoms and possibly causes of several neurodegenerative diseases, including Alzheimer's disease, Parkinson's disease, and amyotrophic lateral sclerosis $(\mathrm{ALS})^{10}$. For instance, in ALS mice, one of the earliest observed pathologies is a decrease in retrograde transport ${ }^{11}$ that eventually leads to axon degeneration. If external forces can modulate active transport of vesicles in neurons, it may be possible to apply tension to ALS axons (via biochemical or mechanical manipulation) to increase active transport as well as induce a retrograde bias to restore normal axonal transport and prevent degeneration. However, to understand this process it is necessary to study different types of neurons and their sensitivity to strain rate and magnitude.

In neurons, the basis of synaptic plasticity is a change in synaptic strength in response to stimuli, and it is believed to be a critical process in mediating the storage of memory in animals ${ }^{27}$. Synaptic plasticity is partially regulated by the amount of neurotransmitter released at the presynaptic terminal ${ }^{28}$. Neurotransmitter release is a cyclic process involving transport of vesicles to active zones, clustering of vesicles at the presynaptic terminal, exocytosis of neurotransmitters via vesicle fusion, and retrieval and recycling of vesicle membrane after release $e^{7,8}$. Mechanical tension plays a role in many of these processes. Neurons regulate their membrane tension ${ }^{29,30}$, and increased tension increases the probability of exocytosis and release $^{31-33}$. Axonal tension is critical in maintaining clustering of vesicles at the presynaptic terminal, and axonal stretching leads to a further increase in vesicle clustering ${ }^{15,16}$. It is hypothesized that increased tension leads to actin polymerization in the synapse, which results in greater actin-synapsin binding sites for vesicles to remain localized at the presynaptic terminal ${ }^{9,34,35}$. However, with increased neurotransmitter release under tension ${ }^{36,37}$, the available supply of vesicles may become depleted. Our results indicate that neuronal tension modulates the active transport of vesicles in neurons by increasing activity of molecular motors and increasing the effective self-diffusivity of the cargo. Interestingly, this process occurs on the same timescale as synaptic potentiation in Aplysia neurons ${ }^{9}$. This is a plausible mechanism to increase delivery of vesicles (containing neurotransmitters and other synaptic machinery) to the presynaptic zone to provide the building blocks for structural plasticity, increase the available pool of neurotransmitters, and maintain a sufficient supply during elevated release. The emerging scenario is that mechanical tension in neurons increases vesicle transport, presynaptic clustering, and neurotransmission; all of which are critical in the process of synaptic plasticity.

\section{Conclusion}

Our results suggest that mechanical stretch leads to increased active transport of vesicles in neurons. The increase in active transport could contribute to tension dependent presynaptic clustering ${ }^{15,16}$, and support tension induced neurotransmission ${ }^{36,37}$. Neurons regulate their internal tension and this may be a key element in maintaining proper function. Thus, mechanical tension could serve as a robust and simple signal to coordinate biophysical processes critical to neuronal function.

\section{Methods}

Cell culture. Aplysia bag cell neurons were isolated and cultured as in previous studies $^{38}$. A. californica were obtained from the National Resource for Aplysia (University of Miami/RSMAS, Miami, FL, USA). Neurons were mechanically dissociated from the Aplysia CNS after a 30-60 min incubation in 1\% protease. Cells were plated in artificial sea water antibiotic solution on fibronectin coated PDMS substrates at room temperature for $12 \mathrm{~h}$ and then at $14^{\circ} \mathrm{C}$ for $24-48 \mathrm{~h}$. Aplysia cells exhibited highly polarized outgrowth exhibiting long straight neurites. Experiments were conducted with cells from multiple animals $(n>19)$ and multiple vesicles $(n>$ 200) were tracked for each cell.

Cell stretching. Neurons were stretched using a system developed for high-resolution live-imaging of cells under applied mechanical strain ${ }^{17}$. The system applied a static deformation to a stretchable polydimethylsiloxane (PDMS) cell culture substrate. In this study, cells were stretched $(20 \%)$ or compressed $(8 \%)$ for $40 \mathrm{~min}$ and then the deformation was removed. All time points prior to $t=0 \mathrm{~min}$ are internal controls. The substrate deformation was characterized experimentally by digital image correlation and computationally by the finite element method. It was found that the mechanical strain of the cell culture surface was uniform over greater than $95 \%$ of the surface area. The details of the stretching system have been previously published ${ }^{17}$.

Microscopy and image analysis. All images were collected on an Olympus IX81 using a (1.15 NA) water immersion objective lens (Olympus America, Center Valley, PA, USA). An Andor Neo sCMOS camera cooled to $-30^{\circ} \mathrm{C}$ was used to record images (Andor Technology, Belfast, UK). All imaging parameters (e.g., light intensity, exposure time, gain, etc.) were kept constant during all experiments. Differential interference contrast (DIC) image sequences of neurons were captured at a rate of 200 frames per second. Videos were captured approximately every $5 \min (n>20$ videos 
$\tau=0.15 \mathrm{~s}$
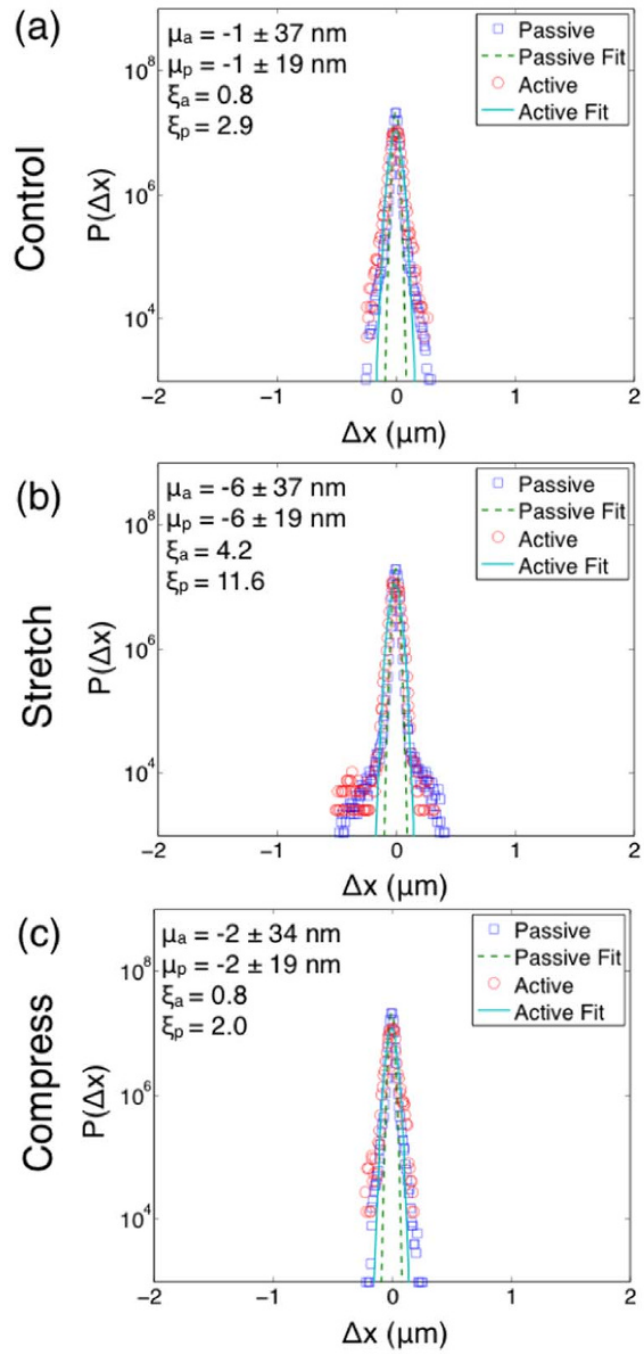

$\tau=1.0 \mathrm{~s}$
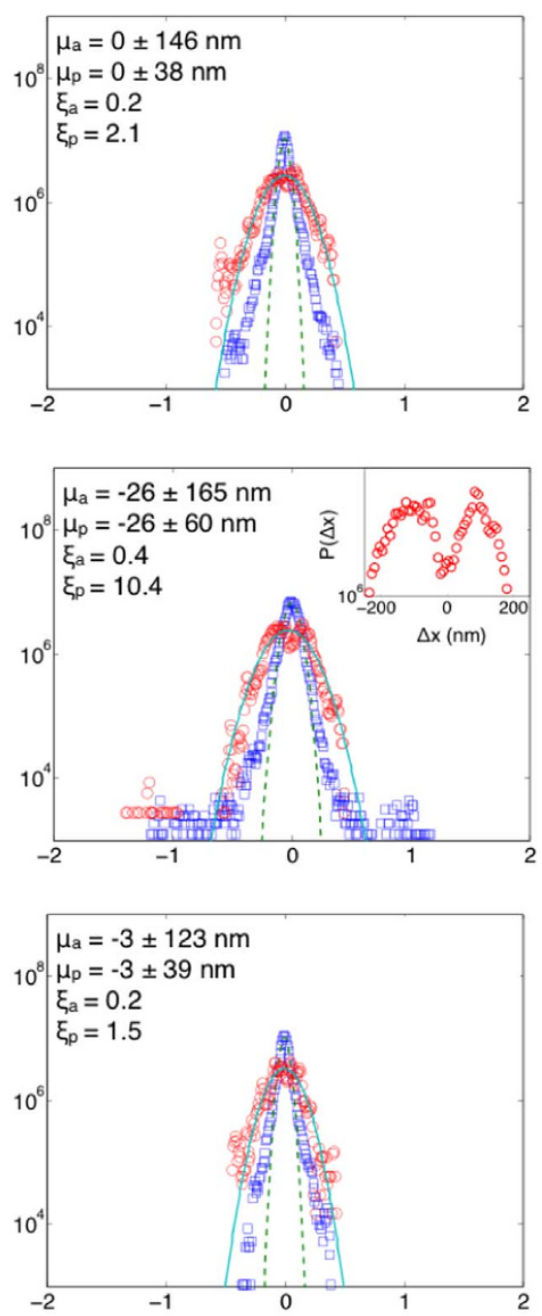

$\tau=5.0 \mathrm{~s}$
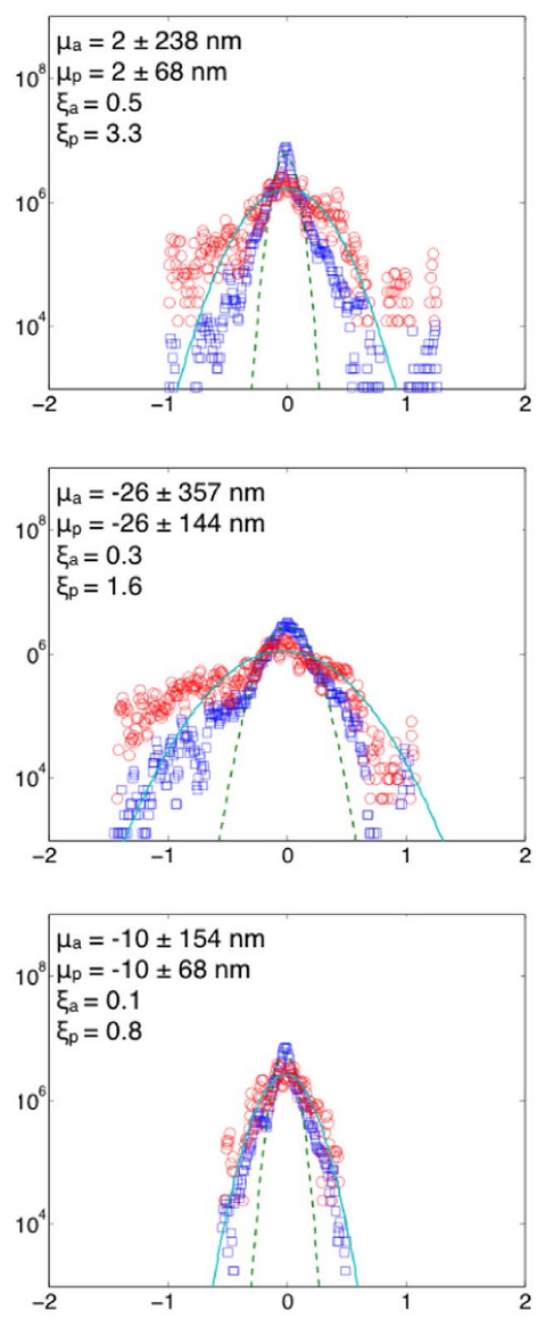

Figure $5 \mid$ Molecular motor activity increases due to stretch. Here $\mu=\bar{x} \pm \sigma$, where $\bar{x}$ is the mean and $\sigma$ the standard deviation of the Gaussian fit, and $\xi$ is the non-Gaussian parameter. The subscript "a" indicates data for active vesicles and "p" are passive vesicles. (a) Vesicles in control neurons exhibit no significant bias $(\bar{x} \approx 0 \mathrm{~nm})$ and small values of the $\xi$ for both active and passive vesicles at all timescales. (b) When neurons are stretched the activity of vesicles increases. At short timescales $(\tau=0.15 \mathrm{~s}), \xi$ increases for active and passive vesicles, indicating an increase in activity possibly due to single molecular motors. At intermediate timescales $(\tau=1 \mathrm{~s}), \sigma_{\text {stretch }}>\sigma_{\text {control }}$ indicating an increase in the activity of collective molecular motor ensembles, and $\bar{x}_{\text {stretch }}=-26 \mathrm{~nm}$ suggesting a retrograde bias of vesicle motion. The zoomed inset shows the local minima in the distribution indicating a lower probability for a vesicle to stay stationary. At large timescales $(\tau=5 \mathrm{~s}), \xi$ values are low suggesting little influence from single motor activity and $\mu$ values remain large suggesting increased activity of collective motors and a retrograde bias. The probability of retrograde motion of active vesicles was estimated as $p_{\mathrm{r} \text {,stretch }}(\tau=5 \mathrm{~s})=0.59$ whereas for all other cases no bias was observed ( $\left.p_{\mathrm{r}} \approx 0.5\right)$. (c) When neurons were compressed, vesicle activity decreased overall for all timescales as indicated by decreased values of $\sigma_{\text {compress }}$ and $\xi_{\text {compress }}$ in comparison to the control.

per cell). Image stack alignment to remove drift was completed with a subpixel registration algorithm ${ }^{39}$. Large dense core vesicle motion was tracked using an algorithm for precise particle tracking by polynomial fitting with Gaussian weight ${ }^{40}$ in MATLAB (The MathWorks, Natick, MA, USA). Vesicle radius is estimated via inflection points in the image intensity profile ${ }^{40}$. All computations, statistical analysis, and plots were generated in MATLAB.

Temporal mean squared displacement analysis. To discriminate between active and passive motion, vesicle dynamics were analyzed using a temporal Mean Squared Displacement $(t \mathrm{MSD})$ algorithm to extract the local behavior of the vesicle as a function of time ${ }^{6}$. The algorithm estimates the local behavior for time, $t$, by calculating the $t \mathrm{MSD}$ of a rolling window centered about the time of interest,

$$
t \operatorname{MSD}(\tau)=\left\langle\left|\mathbf{r}\left(t^{\prime}+\tau\right)-\mathbf{r}\left(t^{\prime}\right)\right|^{2}\right\rangle_{\left(t-\tau_{\max } / 2\right)<t^{\prime}<\left(t+\tau_{\max } / 2\right)}
$$

where $\langle\ldots\rangle$ indicates an average over timescale $\tau, \mathbf{r}(t)$ is the position vector as a function of time, $t^{\prime}$ is the time in the rolling window, $\tau$ is the timescale, and $\tau_{\max }$ is the width of the rolling window $\left(\tau_{\max }=500 \mathrm{~ms}\right)$. The $t$ MSD is then fitted to a power law of the form, $t \operatorname{MSD}(\tau)=C \tau^{\alpha}$ on the interval $100-160 \mathrm{~ms}$, and the power law scaling, $\alpha(t)$, can be extracted as a function of time to indicate the type of diffusion the vesicle is undergoing $(\alpha<1$, subdiffusive; $\alpha=1$, Brownian; $\alpha>1$, superdiffusive). It should be noted that MSD analyses can be highly sensitive to noise and should be interpreted carefully ${ }^{41,42}$. Previously it has been shown in Aplysia neurons that $\alpha \geq 1.4$ is indicative of active motion driven via molecular motors, and $\alpha<1.4$ is considered passive motion $^{6}$. By convention, positive values indicate direction forward towards the neurite terminal (anterograde) and negative values are rearward (retrograde) towards the cell body. This method was used to determine if vesicles were undergoing active or passive motion as used throughout the study.

1. Barlan, K., Rossow, M. J. \& Gelfand, V. I. The journey of the organelle: teamwork and regulation in intracellular transport. Curr. Opin. Cell Bio. 25, 1-6 (2013).

2. Frey, E. Physics in cell biology: on the physics of biopolymers and molecular motors. Chem Phys Chem 3, 270-275 (2002).

3. Brangwynne, C. P., Koenderink, G. H., MacKintosh, F. C. \& Weitz, D. A. Intracellular transport by active diffusion. Trends in Cell Bio. 19, 423-427 (2009).

4. Hirokawa, N. \& Takemura, R. Molecular motors and mechanisms of directional transport in neurons. Nat. Rev. Neur. 6, 201-214 (2005). 
5. Arcizet, D., Meier, B., Sackmann, E., Rädler, J. \& Heinrich, D. Temporal analysis of active and passive transport in living cells. Phys. Rev. Let. 101, 248103 (2008).

6. Ahmed, W. W., Williams, B. J., Silver, A. M. \& Saif, T. A. Measuring nonequilibrium vesicle dynamics in neurons under tension. Lab Chip 13, 570-578 (2013).

7. Owald, D. \& Sigrist, S. J. Assembling the presynaptic active zone. Curr. Opin. Neurobio. 19, 311-318 (2009).

8. Hawkins, R. D., Kandel, E. R. \& Siegelbaum, S. A. Learning to modulate transmitter release: themes and variations in synaptic plasticity. Ann. Rev. Neur 16, 625-665 (1993).

9. Jin, I., Udo, H. \& Hawkins, R. D. Rapid increase in clusters of synaptophysin at onset of homosynaptic potentiation in Aplysia. Proc. Natl. Acad. Sci. USA 108, 11656-11661 (2011).

10. De Vos, K. J., Grierson, A. J., Ackerley, S. \& Miller, C. C. J. Role of axonal transport in neurodegenerative diseases. Ann. Rev. Neur. 31, 151-173 (2008).

11. Bilsland, L. G. et al. Deficits in axonal transport precede ALS symptoms in vivo. Proc. Natl. Acad. Sci. USA 107, 20523-20528 (2010).

12. Lamoureux, P., Buxbaum, R. E. \& Heidemann, S. R. Direct evidence that growth cones pull. Nature 340, 159-162 (1989).

13. Heidemann, S. R. \& Buxbaum, R. E. Tension as a regulator and integrator of axonal growth. Cell Mot. Cytoskel. 17, 6-10 (1990).

14. Zheng, J. et al. Tensile regulation of axonal elongation and initiation. J. Neurosci. 11, 1117-1125 (1991).

15. Siechen, S., Yang, S., Chiba, A. \& Saif, M. T. A. Mechanical tension contributes to clustering of neurotransmitter vesicles at presynaptic terminals. Proc. Natl. Acad. Sci. USA 106, 12611-12616 (2009).

16. Ahmed, W. W. et al. Mechanical tension modulates local and global vesicle dynamics in neurons. Cell. Mol. Bioeng. 5, 155-164 (2012).

17. Ahmed, W. W., Kural, M. \& Saif, T. A. A novel platform for in situ investigation of cells and tissues under mechanical strain. Acta Biomat. 6, 2979-2990 (2010).

18. Burmeister, D. W., Chen, M., Bailey, C. \& Goldberg, D. J. The distribution and movement of organelles in maturing growth cones: correlated video-enhanced and electron microscopic studies. J. Neurocytology 17, 783-795 (1988).

19. Stuhrmann, B., Soares e Silva, M., Depken, M., MacKintosh, F. \& Koenderink, G. Nonequilibrium fluctuations of a remodeling in vitro cytoskeleton. Phys. Rev. E 86, 020901 (2012).

20. Boon, J. P. \& Yip, S. Molecular Hydrodynamics. McGraw-Hill Inc. (1980)

21. Höfling, F. \& Franosch, T. Anomalous transport in the crowded world of biological cells. Rep. Prog. Phys. 76, 046602 (2013).

22. Toyota, T., Head, D. A., Schmidt, C. F. \& Mizuno, D. Non-Gaussian athermal fluctuations in active gels. Soft Matter 7, 3234 (2011).

23. Loverdo, C., Bénichou, O., Moreau, M. \& Voituriez, R. Enhanced reaction kinetics in biological cells. Nat. Phys. 4, 134-137 (2008).

24. Franze, K. \& Guck, J. The biophysics of neuronal growth. Rep. Prog. Phys. 73, 094601 (2010)

25. Suter, D. M. \& Miller, K. E. The emerging role of forces in axonal elongation. Prog. Neurobio 94, 91-101 (2011).

26. Ahmed, W. W., Rajagopalan, J., Togangchi, A. \& Saif, T. A. Neuromechanics: The Role of Tension in Neuronal Growth and Memory. Nano Cell Mech. 3, (2012)

27. Martin, S. J., Grimwood, P. D. \& Morris, R. G. Synaptic plasticity and memory: an evaluation of the hypothesis. Ann. Rev. Neur. 23, 649-711 (2000).

28. Park, Y. \& Kim, K.-T. Short-term plasticity of small synaptic vesicle (SSV) and large dense-core vesicle (LDCV) exocytosis. Cell. Signal. 21, 1465-1470 (2009).

29. Dai, J., Sheetz, M. P., Wan, X. \& Morris, C. E. Membrane tension in swelling and shrinking molluscan neurons. J. Neurosci. 18, 6681-6692 (1998).

30. Pfenninger, K. H. Plasma membrane expansion: a neuron's Herculean task. Nat. Rev. Neurosci. 10, 251-261 (2009).

31. Gauthier, N. C., Fardin, M. A., Roca-Cusachs, P. \& Sheetz, M. P. Temporary increase in plasma membrane tension coordinates the activation of exocytosis and contraction during cell spreading. Proc. Natl. Acad. Sci. USA 108, 14467-14472 (2011).

32. Shillcock, J. \& Lipowsky, R. Tension-induced fusion of bilayer membranes and vesicles. Nat. Mat. 4, 225-228 (2005).
33. Zhu, Y. \& Stevens, C. F. Probing synaptic vesicle fusion by altering mechanical properties of the neuronal surface membrane. Proc. Natl. Acad. Sci. USA 105 18018-18022 (2008).

34. Cingolani, L. A. \& Goda, Y. Actin in action: the interplay between the actin cytoskeleton and synaptic efficacy. Nat. Rev. Neurosci. 9, 344-356 (2008).

35. Morales, M., Colicos, M. A. \& Goda, Y. Actin-dependent regulation of neurotransmitter release at central synapses. Neuron 27, 539-550 (2000).

36. Chen, B. M. \& Grinnell, A. D. Integrins and modulation of transmitter release from motor nerve terminals by stretch. Science 269, 1578-1580 (1995).

37. Grinnell, A. D. et al. The role of integrins in the modulation of neurotransmitter release from motor nerve terminals by stretch and hypertonicity. J. Neurocyt. 32, 489-503 (2003)

38. Romanova, E. V., Oxley, S. P., Rubakhin, S. S., Bohn, P. W. \& Sweedler, J. V. Selfassembled monolayers of alkanethiols on gold modulate electrophysiological parameters and cellular morphology of cultured neurons. Biomaterials 27, 1665-1669 (2006).

39. Thevenaz, P., Ruttimann, U. \& Unser, M. A pyramid approach to subpixel registration based on intensity. IEEE Trans. Image Proc. 7, 27-41 (1998).

40. Rogers, S., Waigh, T., Zhao, X. \& Lu, J. Precise particle tracking against a complicated background: polynomial fitting with Gaussian weight. Phys. Bio. 4, 220-227 (2007)

41. Savin, T. \& Doyle, P. S. Static and dynamic errors in particle tracking microrheology. Biophys. J. 88, 623-638 (2005).

42. Savin, T. \& Doyle, P. S. Role of a finite exposure time on measuring an elastic modulus using microrheology. Phys. Rev. E 71, 041106 (2005).

43. Hendricks, A. G. et al. Dynein Tethers and Stabilizes Dynamic Microtubule PlusEnds. Curr. Bio. 22, 632-637 (2012)

44. Tang-Schomer, M. D., Patel, A. R., Baas, P. W. \& Smith, D. H. Mechanical breaking of microtubules in axons during dynamic stretch injury underlies delayed elasticity, microtubule disassembly, and axon degeneration. FASEB J. 24, 1401-1410 (2010).

45. Vandorpe, D. H. \& Morris, C. E. Stretch Activation of the Aplysia S-Channel. J. Mem. Bio. 127, 205-214 (1992).

\section{Acknowledgments}

The authors thank Prof. E. Sackmann, Prof. J. Rajagopalan and Dr. E. De Souza for fruitful discussions, S.E. Leon and B.W. Williams for assistance in MATLAB computation, and Dr. S. Rubakhin and X. Wang for preparation of neuronal cultures. W.W. Ahmed thanks Frederic T. and Edith F. Mavis for generous support via the MF3 program. This work was supported by the National Science Foundation (CMMI 0800870, ECCS 0801928, CBET 0939511).

\section{Author contributions}

W.W.A. and T.A.S. designed research; W.W.A. developed the experimental tools and analytic techniques; W.W.A. performed research; W.W.A. and T.A.S. analyzed data; and W.W.A. and T.A.S. wrote the paper.

\section{Additional information}

Supplementary information accompanies this paper at http://www.nature.com/ scientificreports

Competing financial interests: The authors declare no competing financial interests.

How to cite this article: Ahmed, W.W. \& Saif, T.A. Active transport of vesicles in neurons is modulated by mechanical tension. Sci. Rep. 4, 4481; DOI:10.1038/srep04481 (2014).

cc)(i) (2) This work is licensed under a Creative Commons Attribution-

BY NC SA NonCommercial-ShareAlike 3.0 Unported license. To view a copy of this license, visit http://creativecommons.org/licenses/by-nc-sa/3.0 